\title{
Omitting age-dependent mosquito mortality in malaria models underestimates the effectiveness of insecticide-treated nets
}

Melissa A. Iacovidou ${ }^{*}, 2$, Priscille Barreaux ${ }^{3}$, Matthew B. Thomas ${ }^{4}$, Erin E. Gorsich $^{2,5}$, Kat S. Rock ${ }^{1,2}$

1 Mathematics Institute, University of Warwick, Coventry, United Kingdom 2 The Zeeman Institute for Systems Biology and Infectious Disease Epidemiology Research, University of Warwick, Coventry, United Kingdom 3 Liverpool School of Tropical Medicine, Liverpool, United Kingdom 4 Department of Biology, University of York, York, United Kingdom 5 School of Life Sciences, University of Warwick, Coventry, United Kingdom

*melissa.iacovidou@warwick.ac.uk

\begin{abstract}
Mathematical models of vector-borne infections, including malaria, often assume age-independent mortality rates of vectors, despite evidence that many insects senesce. In this study we present survival data on insecticide-resistant Anopheles gambiae s.l. from field experiments in Côte d'Ivoire. We fit a constant mortality function and two age-dependent functions (logistic and Gompertz) to the data from mosquitoes exposed (treated) and not exposed (control) to insecticidetreated nets (ITNs), to establish biologically realistic survival functions. This enables us to explore the effects of insecticide exposure on mosquito mortality rates, and the extent to which insecticide resistance might impact the effectiveness of ITNs. We investigate this by calculating the expected number of infectious bites a mosquito will take in its lifetime, and by extension the vectorial capacity. Our results show that the predicted vectorial capacity is substantially lower in mosquitoes exposed to ITNs, despite the mosquitoes in the experiment being highly insecticide-resistant. The more realistic age-dependent functions provide a better fit to the experimental data compared to a constant mortality function and, hence, influence the predicted impact of ITNs on malaria transmission potential. In models with age-independent mortality, there is a reduction of $56.52 \%( \pm 14.66)$ for the vectorial capacity under exposure compared to no exposure. However, the two age-dependent functions predicted a larger reduction due to exposure: for the logistic function the reduction is $74.38 \%( \pm 9.93)$ and for the Gompertz $74.35 \%$ $( \pm 7.11)$, highlighting the impact of incorporating age in the mortality rates. These results further show that multiple exposures to ITNs had a considerable effect on the vectorial capacity. Overall, the study highlights the importance of including age dependency in mathematical models of vector-borne disease transmission and in fully understanding the impact of interventions.
\end{abstract}




\section{Author summary}

Interventions against malaria are most commonly targeted on the adult mosquitoes, which transmit the infection from person to person. One of the most important interventions are bed-nets, treated with insecticides. Unfortunately, extensive exposure of mosquitoes to insecticide has led to widespread evolution of insecticide resistance, which might threaten control strategies. Piecing together the overall impact of resistance on the efficacy of insecticide-treated nets is complex, but can be informed by the use of mathematical models. However, there are some assumptions that the models frequently use which are not realistic in terms of the mosquito biology. In this paper, we formulate a model that includes age-dependent mortality rates, an important parameter in vector control since control strategies most commonly aim to reduce the lifespan of the mosquitoes. By using novel data collected using field-derived insecticide-resistant mosquitoes, we explore the effects that the presence of insecticides on nets have on the mortality rates, as well as the difference incorporating age dependency in the model has on the results. We find that including age-dependent mortality greatly alters the anticipated effects of insecticide-treated nets on mosquito transmission potential, and that ignoring this realism potentially overestimates the negative impact of insecticide resistance.

\section{Introduction}

Malaria is a life-threatening vector-borne parasitic disease, which is endemic in 87 countries, mainly in the African Region [1. The World Health Organization's (WHO) "Global Technical Strategy for Malaria 2016-2030" outlines global targets in the fight against malaria, including a $90 \%$ reduction of malaria case incidence by 2030 [2]. Significant progress towards these targets has occurred, where both the number of cases and the number of deaths due to malaria have decreased between 2010-2019, as outlined in a reported published by WHO in 2020 [1]. In 2019, there were around 229 million cases of malaria globally, with $94 \%$ of them being in the African Region. Additionally, during the same year, 409,000 deaths due to malaria have been estimated worldwide.

The vectors responsible for the malaria parasite's transmission, through blood feeding, belong to the Anopheles genus of mosquitoes. The success of the malaria programmes to date is thanks to a range of interventions, most commonly targeted at these vectors. For example, in sub-Saharan Africa around half of the people at risk are sleeping under insecticide-treated nets (ITNs) [1] which are a way to utilise contact pesticides. Between 2000 and 2015, ITNs contributed to the aversion of many cases; $68 \%$ of the cases that were prevented due to any intervention are attributed to ITNs, making them a crucial intervention [3]. Bednets are currently treated with pyrethroids, and there is evidence of an increase in pyrethroid resistance in malaria vectors, which threatens the elimination efforts 1, 4. Hence, due to mutations and natural selection, mosquitoes develop the ability to resist the harmful effects of insecticides, leading to what is called "insecticide resistance" 4. Nevertheless, it is important to evaluate the potential 
impact that insecticide resistance actually has on the efficacy of current malaria interventions [5]. Alout et al. [6] claim that despite insecticide resistance, vector control is still crucial and can be effective against malaria transmission. This is in agreement with the systematic review and meta-analysis by Strode et al. [7], where the authors concluded that ITNs are more effective than untreated bed-nets, despite insecticide resistance.

By targeting mosquitoes, ITNs target the key point in the transmission cycle, not only reducing the opportunity for blood-feeding on humans, but increasing vector-mortality and, therefore, decreasing the number of infectious blood-meals a mosquito will contribute during its life. The use of vector control to reduce or even eliminate infection is well supported by mechanistic transmission models originally developed for malaria by Ross and Macdonald and used extensively ever since [8]. A key metric linked to transmission models is the basic reproduction number, $R_{0}$, which describes the number of secondary cases produced by a single case in an otherwise susceptible population [9]. Garret-Jones [10 took the purely entomological components of $R_{0}$ and named them vectorial capacity. It is defined in [11] as "the expected number of infective mosquito bites that would eventually arise from all the mosquitoes that would bite a single fully infectious person on a single day", i.e. the average number of humans that get infected due to one infectious human per day. These metrics have been used to study the dynamics of vector-borne diseases and also quantitatively assess the possible impact of interventions to control them.

Contact pesticides were being used at the time Macdonald was researching vector control, and that is when he realised that transmission potential was affected by two important factors relating to mosquito longevity [8]:

(a) a mosquito which is infected will only become infectious if it survives the time needed for the pathogen to develop, commonly known as the extrinsic incubation period (EIP), and

(b) once the mosquito is infectious it must take a blood-meal in order to transmit the infection on to a host.

Hence, Macdonald concluded that the number of infectious bites taken by a mosquito will increase the longer the mosquito survives [8,11]. The longer the EIP is, the less chance a mosquito has to survive it, therefore the younger a mosquito is when it gets infected, the more likely it is to transmit the infection. Thus, the transmission potential relies heavily on the survival of the mosquitoes [8]. Due to Macdonald's analysis, many control programmes aim to reduce the lifespan of mosquitoes [8].

The vectorial capacity depends on the mortality rate of the mosquito, which is typically assumed to be age-independent. Studies have suggested that this assumption, namely that mosquitoes do not senesce, may not be realistic enough for transmission models and can underestimate the impact of vector control strategies 12 16. The assumption is often used to simplify, otherwise complex, mathematical models, and not because of its biological relevance [16, 17. On the other hand, it is rare that suitable age-dependent vector mortality data are available to inform more complex models; in addition to average life expectancy 
with and without ITNs in place, the distribution of life expectancy and how this is impacted by intervention is also required. In the present study, we bring more complex mosquito modelling together with detailed field data to demonstrate how we may rethink the way the vectorial capacity is calculated.

To investigate the impact of multiple insecticidal exposures on the mosquitoes and their ability to transmit malaria, we address the following research questions:

1. Does the mortality rate of the mosquitoes change due to insecticide exposure through ITNs?

2. If it does change, how is the vectorial capacity impacted?

3. When considering age-dependent models, how different is the vectorial capacity compared to assuming mortality is age independent?

To answer these, we use data collected from the field in Côte d'Ivoire. Experiments were conducted on field-derived female Anopheles gambiae s.l. mosquitoes, one of the main malaria vectors in Côte d'Ivoire [18]. The setup of the experiments allowed the comparison of the mosquitoes' survival rates when they were exposed to standard ITNs versus when they were exposed to untreated nets. We fitted various survival functions to these data to estimate biologically realistic mosquito mortality rates and used these to obtain the vectorial capacity estimates of the mosquitoes with and without exposure to ITNs. By including realistic survival in the calculation of the vectorial capacity, we can observe how the assumed effectiveness of anti-vectorial interventions is affected.

\section{Materials and methods}

The modelling analysis of the effect on the vectorial capacity with and without the presence of insecticides is conducted using data that were collected in Bouaké, Côte d'Ivoire. Here the focus is on the malaria parasite Plasmodium falciparum, which is one of the six Plasmodium species known to regularly infect humans, and both the most prevalent and deadly parasite in sub-Saharan Africa [19]. We present a detailed outline of the experimental setup, along with a presentation of the data, followed by a breakdown of the computation of the vectorial capacity using these data.

\section{Experimental setup and Data}

All experimental methods were consistent with Penn State IBC protocol no. 48219. The Pennsylvania State University Institutional Review Board determined that the experiments whereby uninfected mosquitoes were attracted to a host did not meet the criteria for human subjects research. The experimental research formed a part contribution to a larger set of studies reviewed and approved by the Côte d'Ivoire Ministry of Health ethics committee (039/MSLS/CNER-dkn), the Pennsylvania State University's Human Research Protection Program under the Office for Research Protections (STUDY00003899 and STUDY00004815).

The experiment was conducted on Anopheles gambiae s.l. at $26 \pm 1^{\circ} \mathrm{C}$ and consisted of two treatments: 
(a) control (non-exposed): in the presence of an untreated net

(b) treated (exposed): in the presence of an ITN

where a one-way tunnel with two cages was used; a 'holding' cage for the mosquitoes and a 'host' cage for the volunteer's foot that was covered with a treated or untreated net (see Fig 1). The mosquitoes in the experiment are considered to be extremely resistant to the pyrethroid deltamethrin, which was used to treat the ITNs. More details regarding the experimental setup are presented further on.

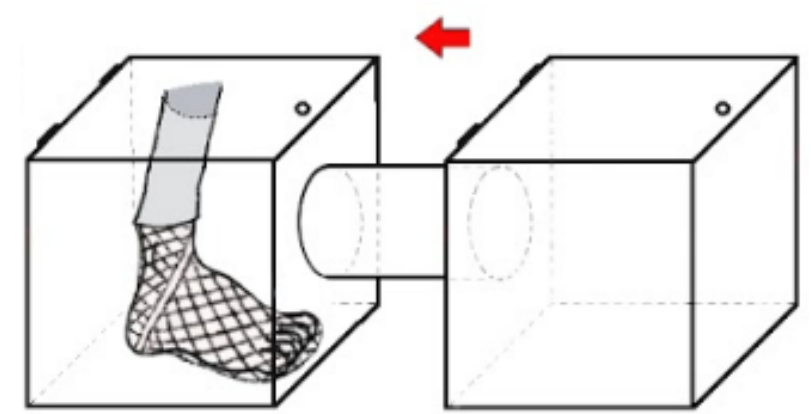

Fig 1. Setup of experiment. The figure depicts the one-way tunnel with the volunteer's foot covered in a net in the 'host' cage on the left, and the 'holding' cage on the right. Both cages are of the same size, $32.5 \times 32.5 \times 32.5 \mathrm{~cm}$. The cages are connected with a PLEXIGLAS $\cap$ tube $(l=30 \mathrm{~cm}, d=14.6 \mathrm{~cm})$. Depending on the chosen treatment for the experiment, the net is either an unwashed PermaNet(R 2.0 or an untreated net, measuring $25 \times 25 \mathrm{~cm}$ in both cases. In this setup the mosquitoes have direct access to the foot for blood feeding.

\section{Mosquito populations}

Anopheles gambiae s.l. mosquitoes were collected as larvae in natural breeding habitats around Bouaké, in central Côte d'Ivoire, and colonised at the Pierre Richet Institute. These are highly pyrethroid-resistant mosquitoes. The 1014F $\mathrm{kdr}$ mutation is almost fixed $(\geq 90 \%)$, and $1575 \mathrm{Y}$, as well as upregulation of CYP6M2, CYP6P3, and CYP9K1 result in a $\geq 1500$-fold resistance to deltamethrin relative to a standard susceptible strain [20]. Larvae were reared at $27 \pm 2^{\circ} \mathrm{C}, 60 \pm 20 \% \mathrm{RH}$ and ambient light in metallic bowls of 300 larvae with $1 \mathrm{~L}$ of deionised water. They were fed daily with TetraMin $\mathrm{R}$ baby fish food following a standardised 'high food' regime as described in Kulma et al. [21]. Adult mosquitoes were kept in $32.5 \times 32.5 \times 32.5 \mathrm{~cm}$ mosquito cages and maintained on $10 \%$ sugar solution. Mosquitoes were four to five days old at their first exposure to insecticide and randomly assigned to a net treatment. Females had constant access to egg laying substrate (a wet cotton pad) and were maintained on a $10 \%$ sugar solution cotton that was renewed daily. Sugar was removed to starve mosquitoes for four hours before each experimental run. 


\section{Human host preparation}

The volunteers involved in this experiment were not actively infected with malaria. They avoided the use of fragrance, repellent products, tobacco, and alcohol for 12 hours before and during testing. For the experiment, feet were washed with unscented soap and rinsed with water the day before a test. The 'host' cages were also washed with soap and rinsed with water every time after a test was conducted - to avoid the accumulation of insecticide particles. Cages were not interchanged between treatments, i.e. a cage used in a control treatment was always used for a control treatment. Note that the data were analysed anonymously.

\section{Insecticide-treated nets (ITNs)}

As mentioned, two types of nets were used: an unwashed PermaNet@ 2.0 (Vestergaard Frandsen SA, DK) and an untreated polyester net (Coghlan's) for the control treatment. The PermaNet(R 2.0 is a long-lasting insecticidal net made of polyester and coated with $55 \mathrm{mg} / \mathrm{m}^{2} \pm 25 \%$ deltamethrin. We confirmed net efficacy by exposing sensitive mosquitoes (Kisumu strain) to WHO tubes lined with a piece of ITN; all mosquitoes died within 24 hours when exposed to the ITN, while the untreated nets killed none. For the wild-type mosquitoes, as these exhibit such a high level of resistance, there was negligible knockdown or mortality $(<1 \%$ knockdown one hour post exposure and no mortality 24 hours later) from ITN exposure in WHO cone assays [22].

\section{Multiple exposure assay}

In malaria-endemic settings with high ITN usage, mosquitoes potentially contact ITNs every time they attempt to feed. To capture this effect, we used a tunnel test in which mosquitoes had to fly a short distance between two cages to locate the host and blood-feed. The tunnel apparatus comprised a standard $32.5 \times 32.5 \times 32.5 \mathrm{~cm}$ mosquito cage as a 'holding' cage, a $32.5 \times 32.5 \times 32.5 \mathrm{~cm}$ mosquito cage as the 'host' cage, and a PLEXIGLASR tube $(l=30 \mathrm{~cm}$, $d=14.6 \mathrm{~cm}$ ) forming the tunnel between cages (as shown in Fig 1). The holding cages were initially populated with 120 pupae each. After adult emergence, 50 females and 10 males were randomly selected to remain in each holding cage until their death, with the excess removed and discarded. We compared the two net treatments, where the foot of a human host was wrapped in a netting sock so the mosquitoes could land on the foot and feed if they chose. Treatments were replicated five times, giving a total of 500 female mosquitoes. Every four days at around $6 \mathrm{pm}$ (dusk) the mosquitoes were exposed to a human foot placed in the 'host' cage. The mosquitoes were allowed to visit the cage for 30 minutes. At the end of 30 minutes, the total number of mosquitoes that had taken a full or partial blood-meal was recorded. The tunnel was then dismantled and all mosquitoes returned to their respective holding cages. The surveillance of the mosquitoes started when they were four days old and tests were repeated every four days until all mosquitoes had died. The number of mosquito deaths was recorded daily. The net treatment was randomly allocated to one human host experimenter to

137 138 139 140 141 142 143 144 146 147 148 149 150 151 152 153 154 155 156 157 158 159 160 161 162 163 164 165 166 167 168 169 170 171 172 173 173
174 174
175 176 177 
ensure there were no biases due to possible differences in attraction between hosts.

During an initial inspection of the data, it was decided that Replicate 1 would be excluded from further analysis. The feeding trend (S1 Fig) of Replicate 1 suggests that the mosquitoes were not feeding and could therefore explain the mortality trend (S2 Fig). The differences in Replicate 1 highlight the fact that mosquito behaviour, for example feeding and host searching, likely depends on a lot of parameters that need further exploration in order to improve transmission models. It seems that there is no significant difference between mosquitoes tested in each treatment in Replicate 1, and this is because they almost never visit the cage with the foot, and thus were not exposed to insecticide during their life. If we were to compare Replicate 1 with the other replicates, it seems probable that blood-meals improve longevity, hence why in both treatments of Replicate 1 the mosquitoes die so early. However, the objective here is to better identify differences of the survival rates between the two treatments, the impact on vectorial capacity, and contrasts when taking age dependencies into account. Since Replicate 1 does not follow the behaviour of the mosquitoes in the rest of the replicates, it is removed from further analysis for consistency.

In the results that follow, the replicates were all combined together, as one larger, aggregated survival dataset, with data from 200 mosquitoes per treatment being examined in total. Fig 2 shows the data used for the calculations. The relevant data can be found in the supporting information (S1 Table, S2 Table S3 Table, S4 Table, S5 Table, and S6 Table).

\section{Control}

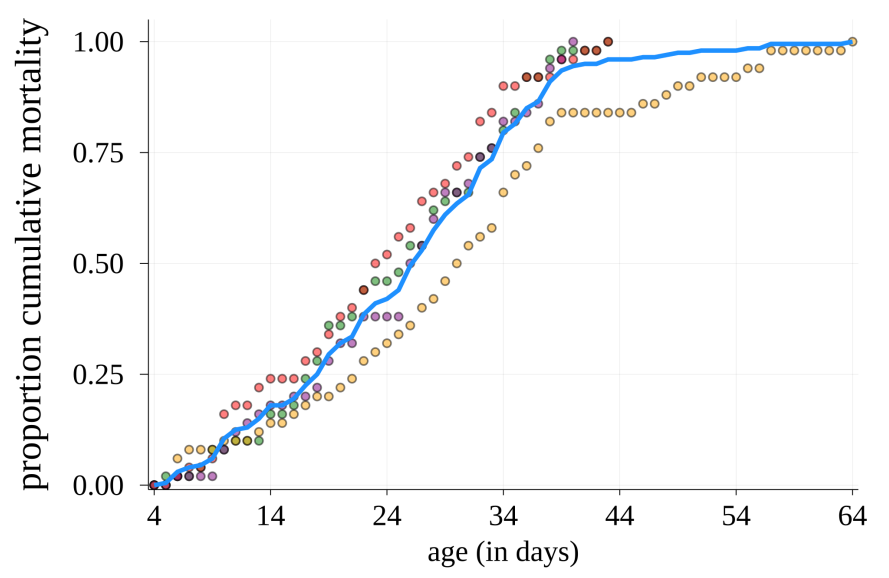

Treated

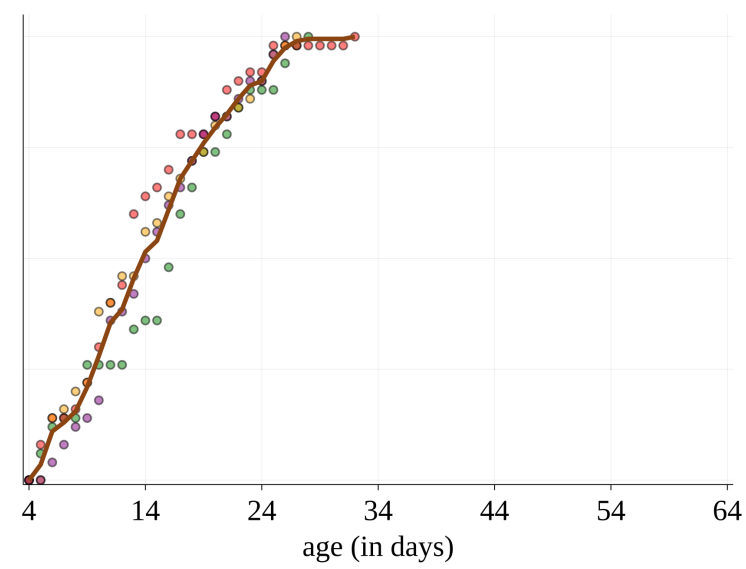

$$
\begin{aligned}
& \text { - } \quad \text { Replicate } 2 \quad \text { - } \quad \text { Replicate } 3 \quad \circ \quad \text { Replicate } 4 \quad \text { • } \quad \text { Replicate } 5 \\
& \longrightarrow \text { Total Control } \longrightarrow \text { Total Treated }
\end{aligned}
$$

Fig 2. Proportion cumulative mortality. The data used for the calculations where all the replicates are combined together. The scatter plots show the cumulative mortality for 50 mosquitoes in each replicate, and the line is the cumulative mortality for 200 mosquitoes in total. Data are plotted up until the final remaining mosquito per replicate died. Replicate 1 was excluded for reasons outlined in the text. 


\section{Vectorial capacity}

Garrett-Jones [10] introduced vectorial capacity, denoted as $C$, in order to estimate the risk of the introduction of malaria. $C$ is commonly defined mathematically as:

$$
C=\frac{m \alpha^{2} p^{n}}{-\ln (p)}
$$

or, equivalently,

$$
C=\frac{m \alpha^{2} e^{-\mu n}}{\mu}
$$

where $m$ is the mosquito density relative to humans, $\alpha$ is the biting rate, $p$ is the survival rate, $\mu$ is the mortality rate, and $n$ is the duration of the EIP, i.e. the number of days between the day a mosquito gets infected until its bites become infectious, and is able to transmit the infection [14].

Eqs (1) and (2) are the two most common forms of the vectorial capacity and are analogous because $\frac{p^{n}}{-\ln (p)}$ is equivalent to $\frac{e^{-\mu n}}{\mu}$, where they both indicate the expectation of a mosquito's infective life, with the former being in respect of the survival probability, and the latter in respect of the mortality rate [23,24. It is important to point out that these forms of the vectorial capacity are predicated on some key assumptions. The first is assuming perfect transmission, although this is easily overcome by a more realistic approach which includes the product $c b$, denoting the vector competence [17,23], hence we have:

$$
C=\frac{m \alpha^{2} c b e^{-\mu n}}{\mu}
$$

A second assumption is that the bite rate is fixed and constant with age. It is often ${ }_{218}$ calculated as the reciprocal of the average length of the gonotrophic cycle [25]. 219 Hence, assuming a gonotrophic cycle of length four, we have $\alpha=0.25$ days $^{-1}$, i.e. $\quad 220$ the mosquitoes feed once every four days, which is in line with the experimental 221 setup, where the mosquitoes were allowed to feed every four days. In our ${ }_{222}$ calculations, we will use $\alpha=0.25$ and keep $m$ and $c b$ as unknown constants. ${ }^{223}$ However, we further investigate the remaining parameters.

\section{Extrinsic incubation period (EIP), $n$}

Another notable assumption is the use of a fixed EIP in Eqs (2) and (3), with the expression $e^{-\mu n}$ representing the probability that a mosquito survives the $n$-day EIP 24]. However, this can be modified to account for non-fixed EIP. A popular choice is to assume the EIP follows an exponential distribution [17]. If the average EIP duration is the same as with the fixed $\operatorname{EIP}(n)$, then the vectorial capacity becomes:

$$
C=\frac{m \alpha^{2} c b}{\mu} \frac{\sigma}{\sigma+\mu}
$$

where $\sigma=\frac{1}{n}$ is the incubation rate. 
As mentioned, the EIP represents the time period where the malaria parasites 233 ingested by the mosquito are developing inside it in order to be able to transmit ${ }_{234}$ the infection. The Centers for Disease Control and Prevention states that $n{ }^{235}$ is at least 9 days, but is dependent on temperature and the different kind of 236 species of the parasites [26]. In a recently published paper by Stopard et al. 27], 237 a mechanistic model fitted to data from 2831 gave an estimate for the median 238 $\left(50^{\text {th }}\right.$ percentile $\left.-\mathrm{EIP}_{50}\right)$, at $27^{\circ} \mathrm{C}$, to be 10.2 days. In addition to the median, 239 the authors provide values for the $10^{\text {th }}$ and $90^{\text {th }}$ percentile $\left(\operatorname{EIP}_{10}\right.$ and $\operatorname{EIP}_{90}, \quad 240$ respectively), so, using these we can calculate a mean value for the EIP for a 241 given distribution at this temperature.

Taking into account the information on the shape of the EIP, it would be more realistic to consider a different distribution which lies somewhere between the exponentially distributed or fixed EIPs. A mathematically neat choice which meets this criteria and is flexible with the addition of a single extra parameter is the Erlang distribution. The Erlang distribution is a special case of the gamma distribution, which has been used for the incubation period in many vector-borne disease models 32 36].

The probability density function (PDF) of the Erlang distribution 37 is given by:

$$
f(x ; k, \lambda)=\frac{\lambda^{k} x^{k-1} e^{-\lambda x}}{(k-1) !}
$$

for $x, \lambda \geq 0$, where $k$ is the shape parameter and $\lambda$ is the rate parameter. The mean is given by $\frac{k}{\lambda}$, which we set equal to $\frac{1}{\sigma}$. Therefore, with $\lambda=k \sigma$ we have:

$$
f(x ; k, \sigma)=\frac{(k \sigma)^{k} x^{k-1} e^{-k \sigma x}}{(k-1) !} .
$$

We match EIP $_{10}$ and EIP $_{90}$ from [27] to our distribution to infer values for $k$ and ${ }_{252}$ $\sigma$. We then take $\lfloor k\rfloor$, since the Erlang distribution requires $k$ to be an integer, ${ }^{253}$ obtaining $k=31$. Using this value for $k$ and the value for $\operatorname{EIP}_{50}$ from [27], we ${ }^{254}$ finally end up with $\sigma=0.097$. Thus, we estimate the mean EIP to be 10.3 days. 255

\section{Mortality rate, $\mu$}

The mortality rate is often assumed to be constant, which drastically simplifies mathematical calculations [16]. Nevertheless, it can be seen from our data that this assumption is not the most realistic. Styer et al. argue in [12] that ignoring mosquito senescence results in inaccurate predictions with respect to vector control effectiveness. For a more realistic approach, we consider an agedependent mortality rate. Henceforth, we consider the following functions for $\mu$ : 
where $a \geq 0$ is the age of the mosquitoes, and the (positive) parameters $\mu_{\text {const }}, \quad 264$ $\mu_{1}, \mu_{2}, \mu_{3}, g_{1}$, and $g_{2}$ are estimated by fitting the data. These functions are often 265 used in demography and population models, since they describe a mortality that 266 increases with age but at a reduced speed 14 .

\section{Results}

Using the data, we need to obtain estimates for the unknown parameters in the mortality functions so that we can calculate the vectorial capacity. In order to investigate the differences in the vectorial capacity between the control and treated cases, and between the various mortality functions, we need to reconsider the way the vectorial capacity is calculated.

\section{Mortality and survival functions}

In order to fit the data, a survival function, $S(a)$, is considered for each mortality function. Its relationship with the mortality function [39] is expressed as:

Using Eq (9), we obtain the survival function for each of the mortality functions: 277

$$
\begin{aligned}
\text { Age-Independent: } & S_{A I}(a)=e^{-a \mu_{\text {const }}} \\
\text { Logistic: } & S_{L}(a)=e^{-\mu_{1} a}\left\{\frac{e^{\mu_{2}\left(\mu_{3}-a\right)}+1}{e^{\mu_{2} \mu_{3}}+1}\right\}^{-\frac{\mu_{1}}{\mu_{2}}} \\
\text { Gompertz: } & S_{G}(a)=e^{-\frac{g_{1}}{g_{2}}\left(e^{a g_{2}}-1\right)}
\end{aligned}
$$

The data for both the control and treated cases are fitted to Eqs (10), (11), and (12). By doing so we obtain the unknown parameters for each function, which are subsequently used in the mortality rate function (Eqs (6), (7), and (8)). The fitting is done using the LsqFit.jl package that provides basic least-squares fitting in Julia [40].

Since the recording of the deaths during the experiment only started when the mosquitoes were already four-days old, we make an assumption, based on observations from the experiments, as to how many mosquitoes were alive at age zero. We formulate two scenarios: the best-case scenario assumes that no mosquitoes died between age zero and four, and the worst-case scenario assumes that there were $1 \%$ more mosquitoes alive initially. Hence, we include an extra "data point" in our dataset. As it turns out, the two scenarios give very close results, so we present the best-case here and the worst-case can be found in the S1 File,

The parameter values obtained are shown in Table 1 and the fitted plots in Fig 3. Note that the error associated with each parameter is propagated through all of the calculations in the Results section using the Measurements.jl package in Julia, employing linear propagation of uncertainty [41. Using the estimated parameter values from Table 1, we plot the mortality functions and compare each treatment (Fig 4). 


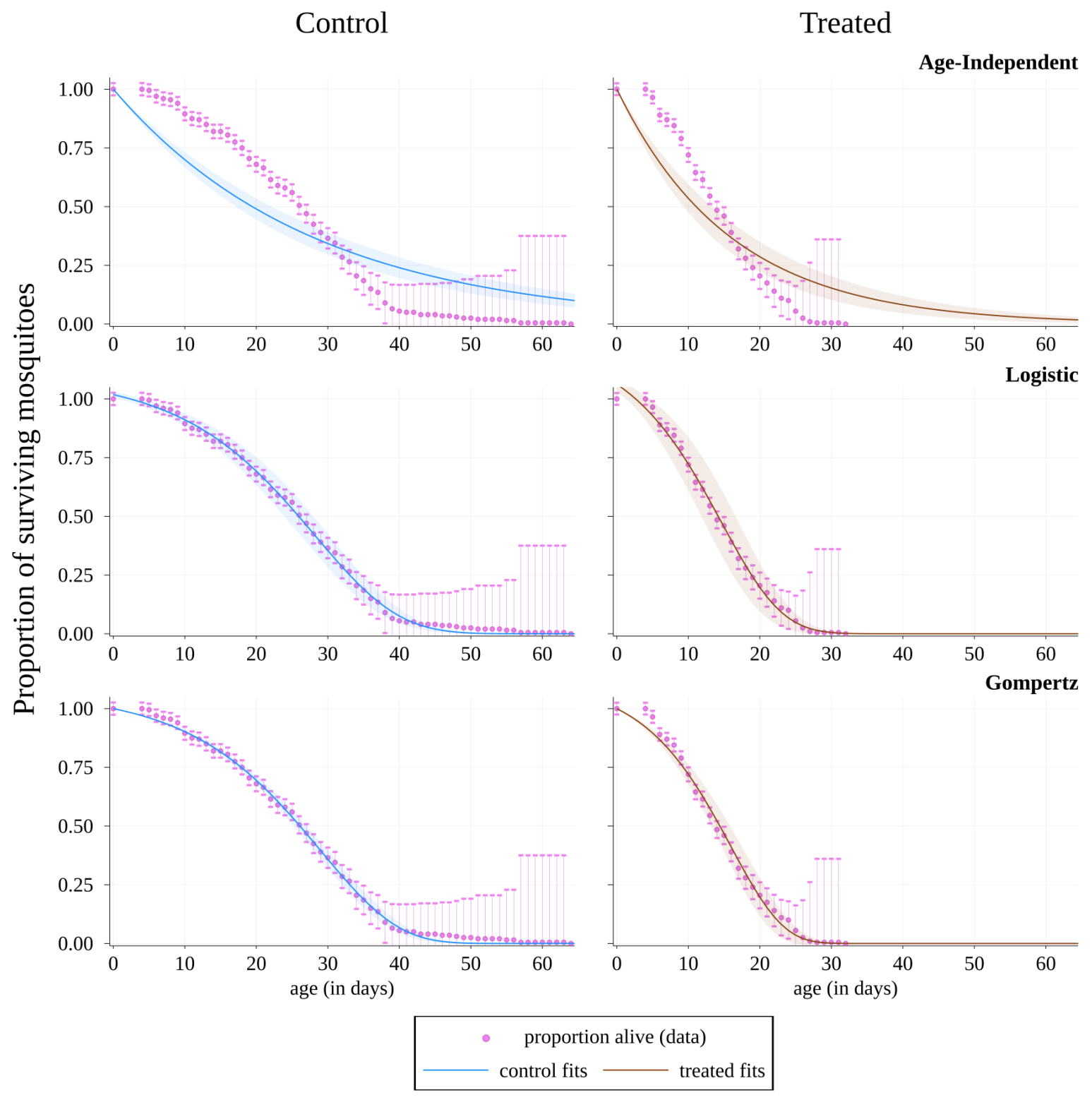

Fig 3. Fitted survival functions. Plots for the proportion of surviving mosquitoes at each day for the control (non-exposed) and treated (exposed) cases using the three different mortality functions (age-independent, logistic, Gompertz). In the treated column, all mosquitoes are dead by day 33. The fits are extended to day 64 for a better comparison between the two treatments. The shaded area represents the error propagated from the parameter estimates in each function. The error bars represent the standard error $\frac{\sigma}{\sqrt{n}}$, where $\sigma$ is the standard deviation of the proportion of alive mosquitoes and $n$ is the number of alive mosquitoes at each point. 

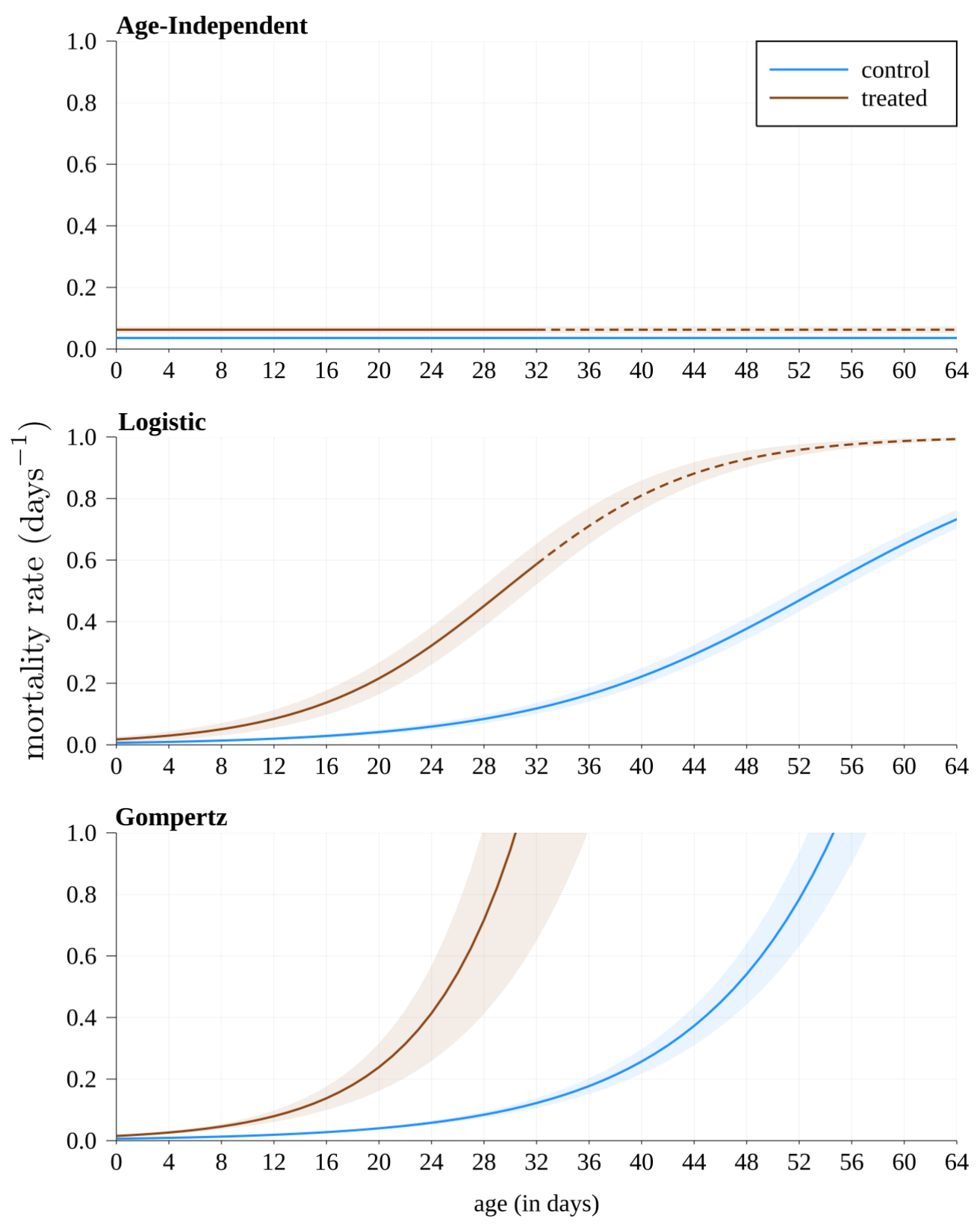

Fig 4. Comparison of mortality for the two treatments. The dashed line represents where all mosquitoes are already dead in the experiment for the treated case. The shaded area represents the error propagated from the parameter estimates in each function. 
Table 1. Fitted parameter values.

\begin{tabular}{|c|c|c|c|c|}
\hline & $\mu_{1}$ & & $\mu_{2}$ & $\mu_{3}$ \\
\hline Control & $1.00 \pm 0.000260$ & 0.0943 & \pm 0.00445 & $53.3 \pm 1.53$ \\
\hline \multirow[t]{2}{*}{ Treated } & $1.00 \pm 0.00102$ & 0.137 & \pm 0.0148 & $29.5 \pm 1.96$ \\
\hline & $g_{1}$ & & $g_{2}$ & $\mu_{\text {const }}$ \\
\hline Control & $0.00625 \pm 0.000447$ & 0.0929 & \pm 0.00347 & $0.0357 \pm 0.00458$ \\
\hline Treated & $0.0153 \pm 0.00244$ & 0.138 & \pm 0.0140 & $0.0625 \pm 0.0110$ \\
\hline
\end{tabular}

The parameter values (3 significant figures) obtained through fitting the three survival functions to the data for the control and treated case, with a margin of error at $5 \%$ confidence level.

\section{Rethinking the vectorial capacity}

We outline the calculations required to obtain the expected number of bites a mosquito takes after being infected and show how this is linked to the vectorial capacity. This will give a more realistic value for $C$. To aid our calculation of the expected number of infectious bites, we pose the following four questions:

1. What is the probability of a mosquito surviving the EIP given an infectious blood-meal is taken at age $a_{0}$ ?

2. How many bites will the mosquito take if it has survived the EIP?

3. What is the expected number of infectious bites a mosquito takes in its lifetime if it has taken an infectious blood-meal at age $a_{0}$ ?

4. What is the expected number of infectious bites a mosquito takes in its lifetime?

Fig 5 depicts a timeline of these events for visualisation purposes. We attempt to answer these questions in four steps. In each step we explore the three different mortality functions: CASE (I) being the age-independent (Eq (6)), CASE (II) the logistic (Eq (7)), and CASE (III) the Gompertz (Eq (8)), and subsequently compare their results.

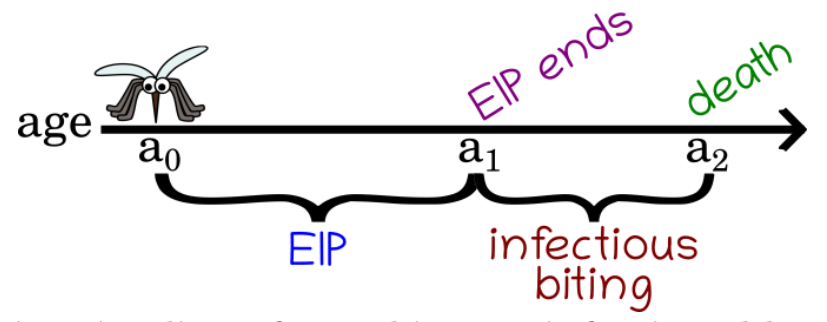

Fig 5. Mosquito timeline after taking an infectious blood-meal. We assume that a mosquito takes an infectious blood-meal at age $a_{0}$. In order for it to become infectious, it must survive the EIP. After surviving the EIP, at age $a_{1}$, the mosquito will take infectious blood-meals up until its death, at $a_{2}$. [Note: the mosquito might not survive the EIP, hence, it is possible that $a_{2}<a_{1}$.] 


\section{Step 1: $\mathbb{P}\left(\right.$ mosquito survives EIP | infectious blood-meal at $\left.a_{0}\right)$}

Using our assumptions for the EIP and the survival of the mosquitoes we calculate the following:

$\mathbb{P}$ (surviving EIP | infectious blood-meal taken at age $a_{0}$ )

$$
\begin{aligned}
& =\int_{t=0}^{\infty} \mathbb{P}(\text { exits EIP after time } t \mid \text { survives to time } t) \\
& \quad \times \mathbb{P}\left(\text { survives from } a_{0} \text { to time } t\right) \mathrm{d} t \\
& =\int_{t=0}^{\infty}[\mathrm{PDF} \text { of } \operatorname{EIP}(t)] \times \exp \left(-\int_{a_{0}}^{a_{0}+t} \mu(x) \mathrm{d} x\right) \mathrm{d} t
\end{aligned}
$$

Using Eq (5):

$$
=\int_{t=0}^{\infty} \frac{(k \sigma)^{k} t^{k-1} e^{-k \sigma t}}{(k-1) !} \times \exp \left(-\int_{a_{0}}^{a_{0}+t} \mu(x) \mathrm{d} x\right) \mathrm{d} t
$$

CASE (I):

$\mathbb{P}$ (surviving EIP | infectious blood-meal taken at age $a_{0}$ )

$$
=\int_{t=0}^{\infty} \frac{(k \sigma)^{k} t^{k-1} e^{-k \sigma t}}{(k-1) !} \times e^{-\mu_{\text {const }} t} \mathrm{~d} t=\left(\frac{k \sigma}{k \sigma+\mu_{\text {const }}}\right)^{k}
$$

CAse (II):

$\mathbb{P}$ (surviving EIP | infectious blood-meal taken at age $\left.a_{0}\right)$

$$
=\int_{t=0}^{\infty} \frac{(k \sigma)^{k} t^{k-1} e^{-k \sigma t}}{(k-1) !} \times e^{-\mu_{1} t}\left\{\frac{e^{\mu_{2}\left(\mu_{3}-\left(a_{0}+t\right)\right)}+1}{e^{\mu_{2}\left(\mu_{3}-a_{0}\right)}+1}\right\}^{-\frac{\mu_{1}}{\mu_{2}}} \mathrm{~d} t
$$

We cannot solve this analytically, but we integrate this numerically using the QuadGK.jl package in Julia.

CAse (III):

$$
\begin{aligned}
& \left.\mathbb{P} \text { (surviving EIP } \mid \text { infectious blood-meal taken at age } a_{0}\right) \\
& =\int_{t=0}^{\infty} \frac{(k \sigma)^{k} t^{k-1} e^{-k \sigma t}}{(k-1) !} \times e^{-\frac{g_{1}}{g_{2}} e^{a_{0} g_{2}}\left(e^{g_{2} t}-1\right)} \mathrm{d} t
\end{aligned}
$$

This is also solved numerically. The results for the three cases are plotted and shown in Fig 6 .

From this first step we notice that the age-independent case is not biologically realistic. Comparing it with the age-dependent cases, we can see that as $a_{0}$ increases, the probability a mosquito will survive the EIP decreases significantly, which makes sense, given that there is evidence mosquitoes senesce both in the data used here, but also from Styer et al. and Ryan et al. 12, 16. We also notice that the Gompertz function's curve approaches zero faster, which could be somewhat more realistic, whereas with the logistic function it seems that we reach a plateau slightly above zero. Comparing the two treatments, control and treated, we notice that the probability is lower initially with treatment, but also that the age-dependent functions approach zero much faster, which is in line with the mosquitoes having a lower life expectancy in the treated case (Fig 3 . 


\section{Control}

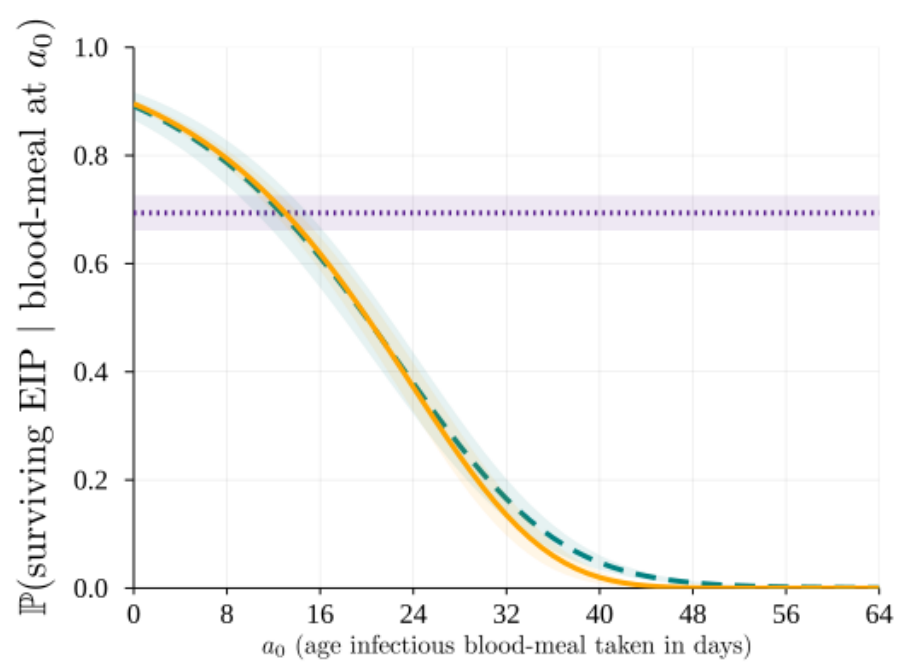

\section{Treated}

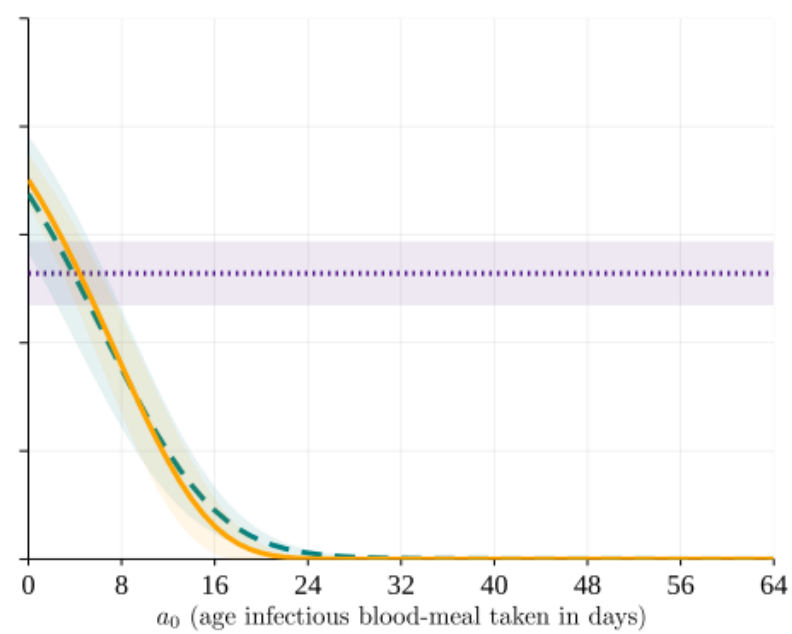

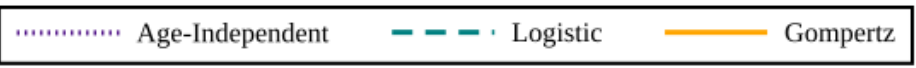

Fig 6. Probability the mosquito survives the extrinsic incubation period given that it takes an infectious blood-meal at age $a_{0}$. The plots show the values of Eqs (13), (14) and (15) over different ages. The shaded area represents the error propagated from the parameter estimates in through the calculations.

Step 2: $\mathbb{P}\left(z=j \mid\right.$ mosquito exits EIP at $\left.a_{1}\right)$

We are interested in the probability mass function (PMF) of the number of bites, $z$, supposing the mosquito exits the EIP at age $a_{1}$, and dies at age $a_{2}$. This is given by:

$$
\begin{array}{r}
\mathbb{P}\left(z=j \mid \text { exits EIP at age } a_{1}\right)=\int_{a_{1}}^{\infty} \mathbb{P}\left(z=j \mid \text { survive from } a_{1} \text { to } a_{2}\right) \\
\times \mathbb{P}\left(\text { dying at } a_{2}\right) \mathrm{d} a_{2}
\end{array}
$$

If we assume that the time between bites are exponentially distributed, the first part is given by a Poisson process of the form:

$$
\frac{\left[\alpha\left(a_{2}-a_{1}\right)\right]^{j}}{j !} e^{-\alpha\left(a_{2}-a_{1}\right)}
$$

and so it remains to calculate the second part. First, we know that the probability of the mosquito surviving between $a_{1}$ and $a^{\prime}$ is equal to $\exp \left(-\int_{a_{1}}^{a^{\prime}} \mu(x) \mathrm{d} x\right)$ (given our assumption it already survived to age $a_{1}$ ). This is equivalent to the probability that the time of death of the mosquito is greater than $a^{\prime}, \mathbb{P}\left(a_{2}>a^{\prime}\right)$. Hence we can write down the cumulative distribution function of the probability the mosquito dies before age $a^{\prime}$ :

$$
F_{a_{2}}\left(a^{\prime}\right)=\mathbb{P}\left(a_{2} \leq a^{\prime}\right)=1-\exp \left(-\int_{a_{1}}^{a^{\prime}} \mu(x) \mathrm{d} x\right)
$$


and so its derivative with respect to $a^{\prime}$ gives us what we require (the probability ${ }_{343}$

of dying at exactly $a^{\prime}$ ):

$$
\frac{\mathrm{d} F_{a_{2}}\left(a^{\prime}\right)}{\mathrm{d} a^{\prime}}=\mu\left(a^{\prime}\right) \exp \left(-\int_{a_{1}}^{a^{\prime}} \mu(x) \mathrm{d} x\right)
$$

Therefore, using Eqs (16) and (17),

$$
\begin{aligned}
& \mathbb{P}\left(z=j \mid \text { exits EIP at age } a_{1}\right)= \\
& \qquad \int_{a_{2}=a_{1}}^{\infty} \frac{\alpha^{j}\left(a_{2}-a_{1}\right)^{j}}{j !} e^{-\alpha\left(a_{2}-a_{1}\right)} \mu\left(a_{2}\right) \exp \left(-\int_{a_{1}}^{a_{2}} \mu(x) \mathrm{d} x\right) \mathrm{d} a_{2}
\end{aligned}
$$

CAse (I):

$$
\mathbb{P}\left(z=j \mid \text { exits EIP at age } a_{1}\right)=\frac{\mu_{\text {const }} \alpha^{j}}{\left(\mu_{\text {const }}+\alpha\right)^{j+1}}
$$

CASE (II):

$$
\begin{aligned}
& \mathbb{P}\left(z=j \mid \text { exits EIP at age } a_{1}\right)= \\
& \qquad \int_{a_{2}=a_{1}}^{\infty} \frac{\alpha^{j}\left(a_{2}-a_{1}\right)^{j}}{j !} e^{-\left(\alpha+\mu_{1}\right)\left(a_{2}-a_{1}\right)} \\
& \frac{\mu_{1}}{1+e^{\mu_{2}\left(\mu_{3}-a_{2}\right)}}\left\{\frac{e^{\mu_{2}\left(\mu_{3}-a_{2}\right)}+1}{e^{\mu_{2}\left(\mu_{3}-a_{1}\right)}+1}\right\}^{-\frac{\mu_{1}}{\mu_{2}}} \mathrm{~d} a_{2}
\end{aligned}
$$

CASE (III):

$$
\begin{aligned}
& \mathbb{P}\left(z=j \mid \text { exits EIP at age } a_{1}\right)= \\
& \qquad \int_{a_{2}=a_{1}}^{\infty} \frac{\alpha^{j}\left(a_{2}-a_{1}\right)^{j}}{j !} e^{a_{2} g_{2}-\left(a_{2}-a_{1}\right) \alpha} g_{1} e^{-\frac{g_{1}}{g_{2}}\left(e^{\left.a_{2} g_{2}-e^{a_{1} g_{2}}\right)}\right.} \mathrm{d} a_{2}
\end{aligned}
$$

CAses (II) and (III) are again solved numerically. We plot some heatmaps to visualise the solutions of Eqs (18), (19), and (20) (Fig 7). In the plots we include the average number of bites, which is calculated using:

$$
\mathbb{E}\left(z \mid \text { exits EIP at age } a_{1}\right)=\sum_{n=0}^{\infty}\left[j \times \mathbb{P}\left(z=j \mid \text { exits EIP at age } a_{1}\right)\right] .
$$

We also plot the PMFs for a specific age $\left(a_{1}=15\right)$ which can be found in S3 Fig, In Fig 7 we can see that, for the age-dependent cases, the older the mosquito is when it exits the EIP, the higher the probability that the number of bites it takes is small. The two age-dependent functions give similar results, but the age-independent function shows again that it is not biologically realistic, since it has a constant average and a constant probability across all ages. In the treated case, their is a higher probability that there are low, or even zero, bites compared to the control case; this happens for all choices of mortality function. 

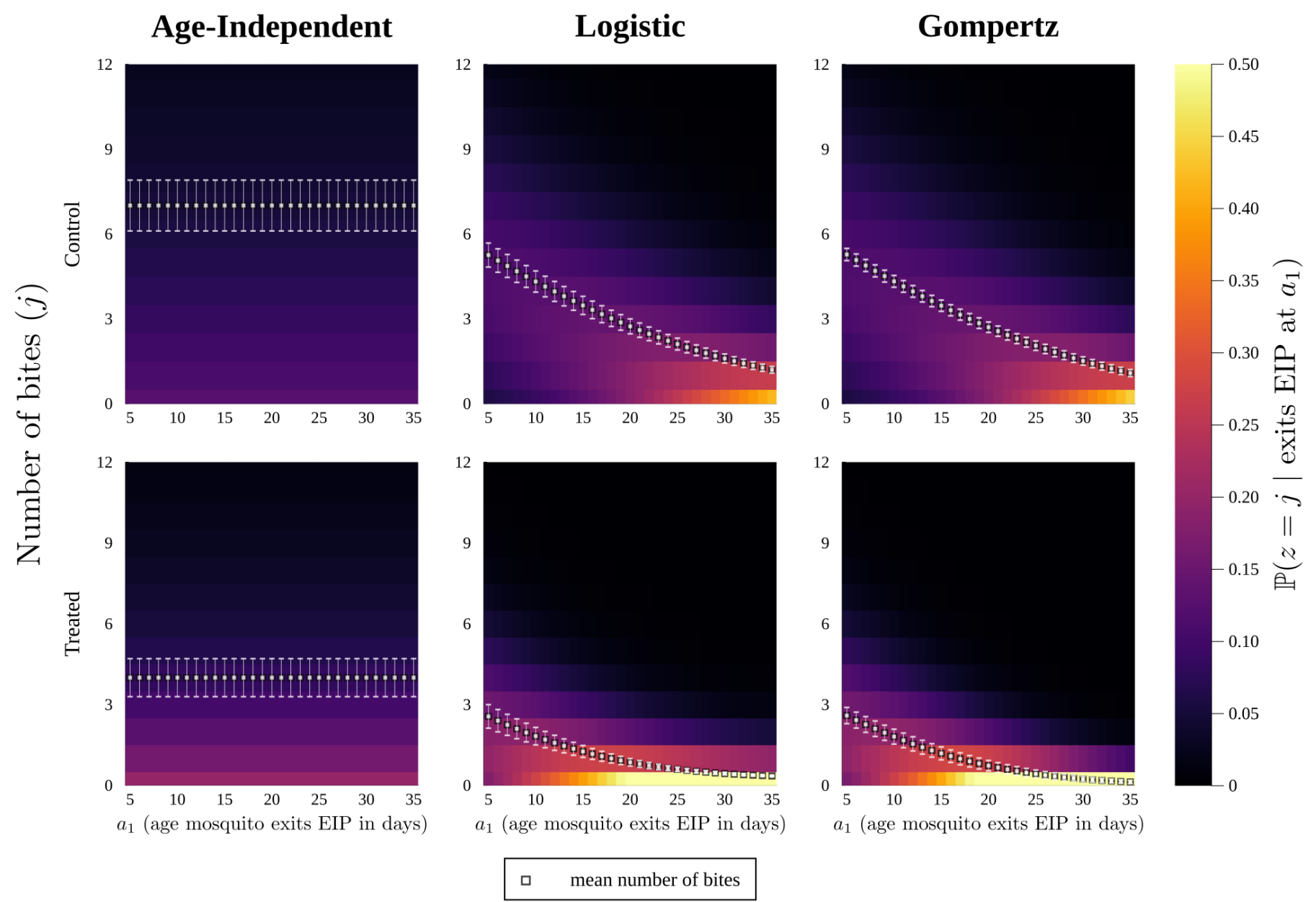

Fig 7. Heatmaps of the probability the number of bites is equal to some $n$ given that the mosquito exits the extrinsic incubation period (EIP) at age $a_{1}$. The error bars on the mean markers represent the propagated uncertainty from the fitted parameters.

Step 3: $\mathbb{E}\left(z \mid\right.$ infectious blood-meal at $\left.a_{0}\right)$

We can now calculate the expected number of infectious bites a mosquito takes, 360 given that it takes an infectious blood-meal at age $a_{0}$ :

$$
\mathbb{E}\left(z \mid \text { blood-meal at } a_{0}\right)=\sum_{j=0}^{\infty}\left[j \times \mathbb{P}\left(z=j \mid \text { blood-meal at } a_{0}\right)\right]
$$

Hence, we need to calculate the PMF of the number of bites, given that an 362 infectious blood-meal is taken at age $a_{0}$. To do so, we use the results from Step 1 and Step 2.

CAse (I): To calculate the probability required, we multiply the results from Eqs (13) and (18), however care must be taken for when $j=0$, where we need to consider that we definitely have zero bites if the mosquito does not survive the EIP. 


$$
\mathbb{P}\left(z=j \mid \text { blood-meal at } a_{0}\right)= \begin{cases}\left(\frac{k \sigma}{k \sigma+\mu_{\text {const }}}\right)^{k} \frac{\mu_{\text {const }} \alpha^{j}}{\left(\mu_{\text {const }}+\alpha\right)^{j+1}} & \text { if } j \neq 0 \\ \left(\frac{k \sigma}{k \sigma+\mu_{\text {const }}}\right)^{k} \frac{\mu_{\text {const }}}{\mu_{\text {const }}+\alpha}+\left(1-\left(\frac{k \sigma}{k \sigma+\mu_{\text {const }}}\right)^{k}\right) & \text { if } j=0\end{cases}
$$

This gives us

$$
\mathbb{E}\left(z \mid \text { infectious blood-meal at } a_{0}\right)=\frac{\alpha}{\mu_{\text {const }}}\left(\frac{k \sigma}{k \sigma+\mu_{\text {const }}}\right)^{k} .
$$

CAses (II) and (III):

$$
\begin{aligned}
& \mathbb{P}\left(z=j \mid \text { blood-meal at } a_{0}\right)= \\
& \left.\int_{a_{1}=a_{0}}^{\infty}[\operatorname{Eq}[19) \text { or Eq } 20)\right] \times \frac{(k \sigma)^{k}\left(a_{1}-a_{0}\right)^{k-1} e^{-k \sigma\left(a_{1}-a_{0}\right)}}{(k-1) !} \\
& \times \exp \left(-\int_{a_{0}}^{a_{1}} \mu(x) \mathrm{d} x\right) \mathrm{d} a_{1}
\end{aligned}
$$

where we use Eq (19) for CASE (II) and Eq (20) for CASE (III). The above is 372 true for $j \neq 0$. When $j=0$, we must add $(1-\mathrm{Eq}(14))$ to Eq (23) for CASE (II), 373 or (1 - Eq (15) for CASE (III), following the same logic as in CASE (I). This 374 is again integrated numerically (this time using the Cuba.jl [42, 43, package in 375 Julia) and put into Eq (21) to obtain the required solution. The results of Step 376 3 are depicted in Fig 8 for both treatments.

The expected number of bites decreases significantly if we consider an agedependent mortality function, as we can see in Fig 8, There is also an obvious difference between the two treatments, where in the treated case the number of bites are a lot lower to begin with. Once again the Gompertz function seems to be tending to zero slightly faster than the logistic, but the difference is negligible.

\section{Step 4: $\mathbb{E}(z)$}

We can now use the previous steps to calculate the expected number of infectious bites a mosquito will take in its lifetime:

$$
\begin{aligned}
\mathbb{E}(z)=\int_{a_{0}=0}^{\infty} \mathbb{E}(z \mid & \text { infectious blood-meal at } \left.a_{0}\right) \\
& \times \mathbb{P}\left(\text { infectious blood-meal at } a_{0}\right) \mathrm{d} a_{0}
\end{aligned}
$$

Breaking this down, the first part is Eq (21), and, for the second part, we must take into consideration that the probability a mosquito gets infected at age $a_{0}$ depends on the PDF of blood-meals. Specifically, we have:

$$
\mathbb{E}(z)=\int_{a_{0}=0}^{\infty} \mathbb{E}\left(z \mid \text { infectious blood-meal at } a_{0}\right) \times \alpha e^{-\alpha a_{0}} \mathrm{~d} a_{0}
$$




\section{Control}

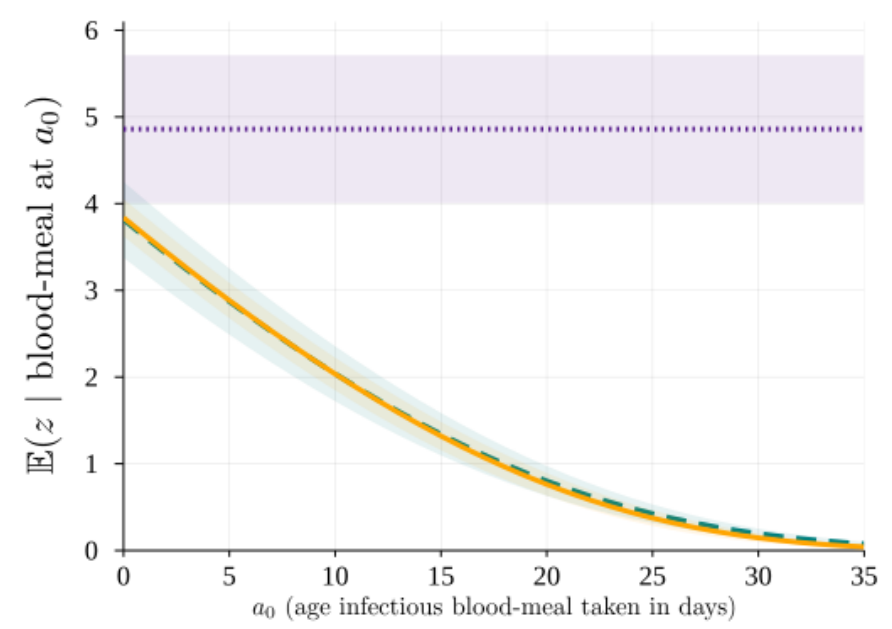

\section{Treated}

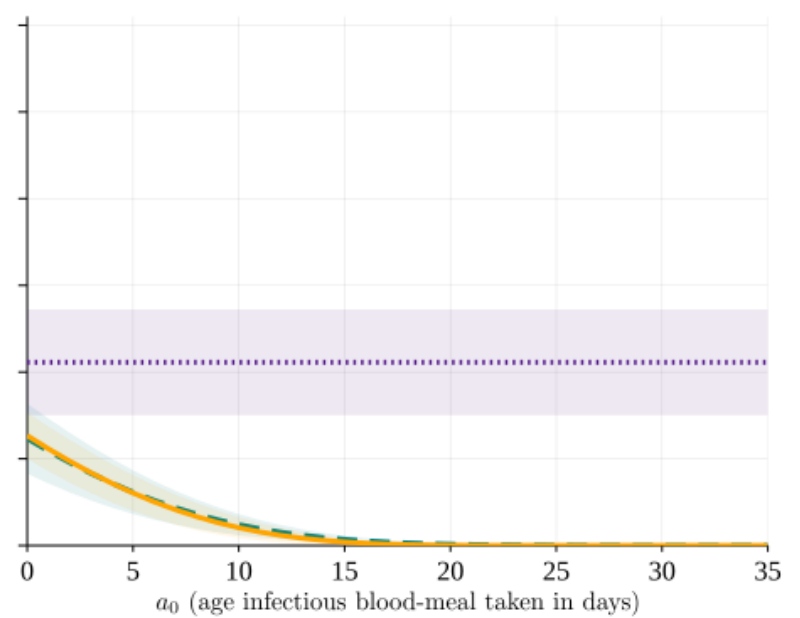

Gompertz

Fig 8. The expected number of bites a mosquito will take in its lifetime given it has taken an infectious blood-meal at age $a_{0}$. The shaded area represents the error propagated from the parameter estimates throughout the calculations.

CASE (I):

$$
\mathbb{E}(z)=\frac{\alpha}{\mu_{\text {const }}}\left(\frac{k \sigma}{k \sigma+\mu_{\text {const }}}\right)^{k}
$$

which, as expected, is the same as Eq $(22)$ as it does not depend on age. Looking 387 back at the vectorial capacity (Eqs (3) and (4)), we can see that this is rep- 388 resented there by $\frac{\alpha}{\mu} e^{-\mu n}$ and $\frac{\alpha}{\mu} \frac{\sigma}{\sigma+\mu}$ respectively. This is because the assumed 389 EIP distribution in each case is different (fixed and exponentially distributed), 390 whereas we have assumed an Erlang distribution.

We solve Eq (24) for CASES (II) and (III) numerically and obtain a single number for each. The results for all cases and both treatments can be found in Fig 9. We observe that the expected number of bites is lower if we consider agedependent mortality functions, where we have $3.09 \pm 0.39$ for the logistic function and $3.10 \pm 0.20$ for the Gompertz, versus $4.86 \pm 0.85$ for the age-independent. With treatment, the numbers are even lower for all cases, where for the age-dependent functions, the expected number of bites is $<1$. Using all of the above, we can calculate the relative difference in the vectorial capacity between the control and treated cases for the age-dependent and age-independent mortality functions. We do so by assuming that the mosquito density, $m$, the vector competence, $c b$, and the bite rate, $\alpha$, are constant. Hence we can use the results from Eq (24) to make our comparisons (Fig 10.

Between the control and treated cases for each function there is a significant reduction in the vectorial capacity, where the percentage for the age-independent 


\section{Control}

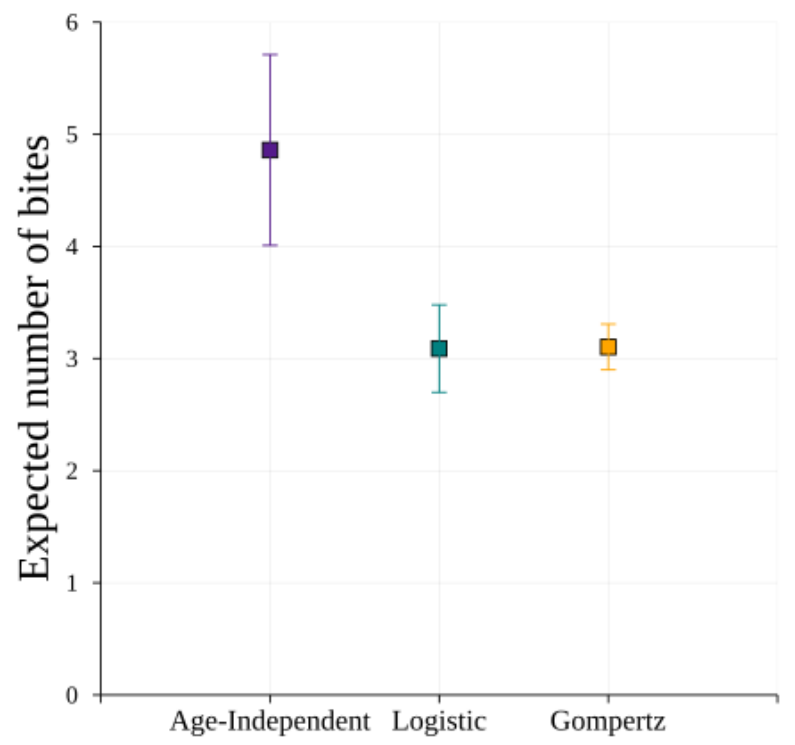

Treated

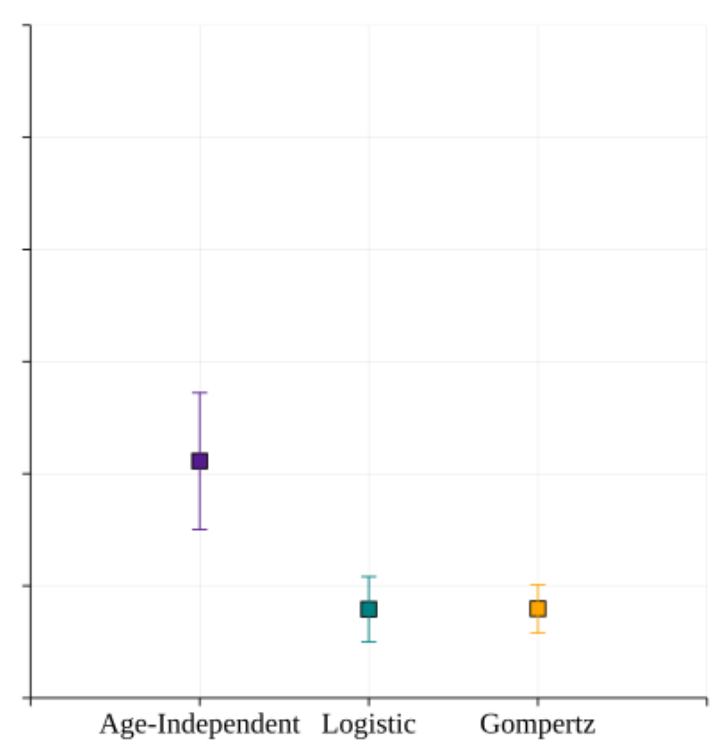

Fig 9. The expected number of infectious bites a mosquito will take in its lifetime. The error bars represent the error propagated from the parameter estimates through the calculations.

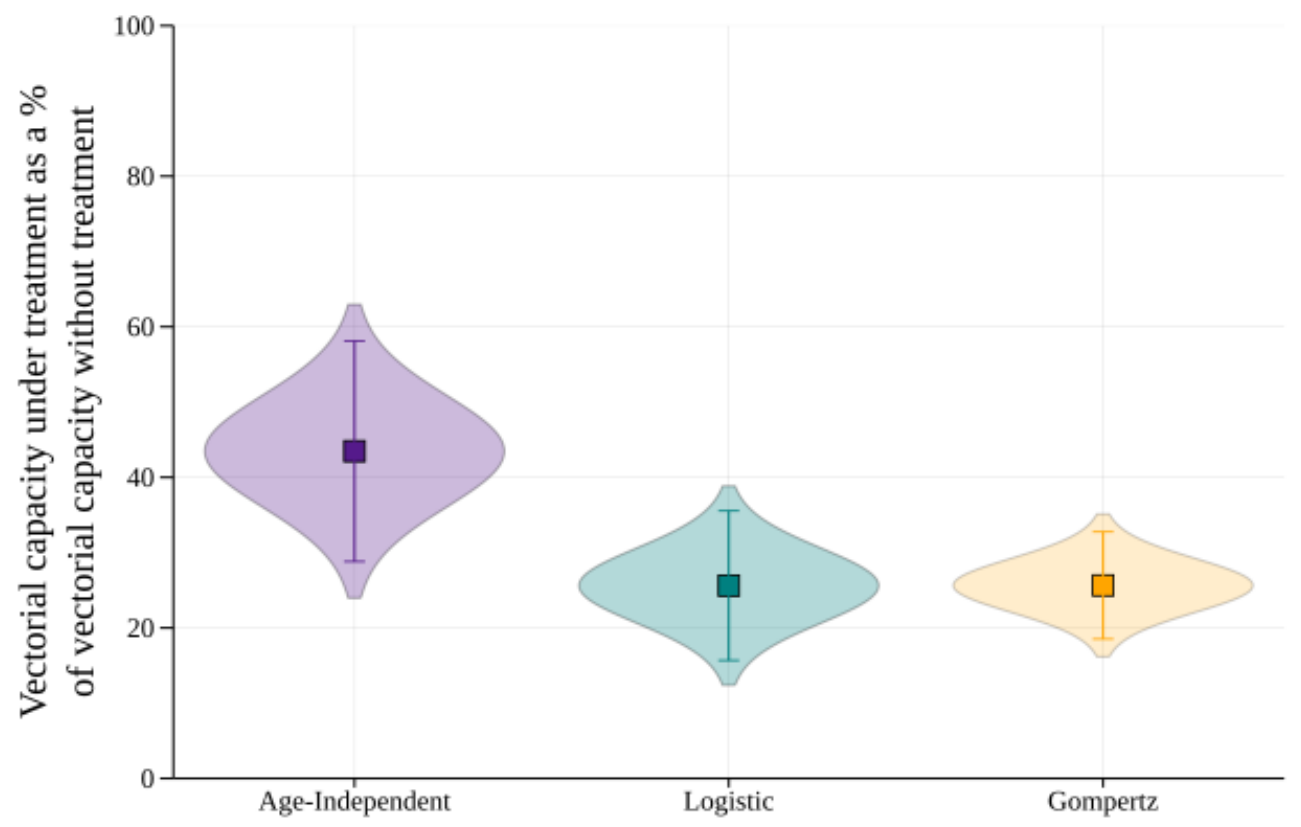

Fig 10. Violin plots showing the relative difference in the vectorial capacity with treatment. The error bars represent the propagated uncertainty due to the margin error of the fitted parameters. 
treated case relative to the control is at $43.5 \%( \pm 14.7)$, or, equivalently, there is a reduction of $56.5 \%( \pm 14.7)$. However, we also notice that the age-dependent functions have a bigger decrease: the difference in the reduction percentages between the age-independent and the logistic cases is $\approx 17.86$ and between the age-independent and the Gompertz cases is $\approx 17.83$. In all previous calculations, we have seen that the age-independent function is not very realistic, whereas the logistic and Gompertz functions behave as we might expect from our understanding of vector senescence. The Gompertz function might be slightly closer to biological realism, however the difference between the two is minimal. Both functions seem to be good candidates, though we might have a stronger preference for using the Gompertz function in future calculations, since it only requires two fitted parameters, compared to three in the logistic function.

406

\section{Discussion}

Mathematical modelling can contribute by recommending improved or optimised intervention strategies for various (vector-borne) diseases, but there are many challenges that modellers have to overcome to provide policy-relevant insights 44 , 45]. One must find a balance between creating easy-to-use models and ensuring that biological simplifications do not alter the resultant policy recommendations by over or underestimating the impact of different intervention measures. One such challenge for modelling malaria is to accurately quantify the mortality of the mosquitoes. In the present study, we have demonstrated that using agedependent mortality functions is important; the EIP is typically long compared to a mosquito's lifespan and combined with senescence this has a substantial impact on our calculation of the vectorial capacity.

This study echos results from other studies (e.g. 14, 15, 17]), which advocate for incorporation of more biological realism in vector-borne disease modelling. Brand et al. [17] consider different distributions for the EIP to show how $R_{0}$ changes. On the other hand, Bellan and Novoseltsev et al., 14, 15 respectively, highlight the importance of age-dependent mortality. In these studies, however, the traditionally fixed EIP is considered, and the calculations for the vectorial capacity are approached from a different perspective. Bellan [14 incorporates a fixed parameter for the impact of insecticides on the longevity of the mosquitoes, due to the lack of real-life data. Similarly, in [15], the authors consider multiple patterns for age-dependent mortality which are generalised for multiple vector species. This highlights the clear need for better availability of real-world data for different vector-parasite systems to improve modelling predictions. We have shown that without accounting for age the effectiveness of the anti-vectorial intervention against highly-pyrethroid-resistant An. gambiae s.l. mosquitoes would be underestimated.

The two age-dependent functions that are explored in this paper are often used for survival analysis $[12,14,15,46]$. Styer et al. investigated a large-scale mortality study using Aedes aegypti mosquitoes and concluded that the logistic mortality functions fit the data better on most occasions except one, where the Gompertz function was a better fit [12]. Clements and Paterson explored 407 408 409 410 411 412 413 414 415 416 417

4
8
9
0
1
2
3
4
4
4


the survival patterns in many different mosquito species and found that their patterns are explained well by using a Gompertz function [46]. Although the logistic and Gompertz are rather flexible functions, other functional forms may be more suitable for different vector species - in this case the general framework presented in here could be readily adapted if required.

The data and results show there is a significant increase in mosquito mortality when several blood-meal opportunities are offered by a host protected with an ITN vs. an untreated net. The consequence for mosquitoes is a reduced vectorial capacity induced by the presence of an insecticide. The fact that standard ITNs do not immediately kill young and unfed mosquitoes following a single and forced exposure to insecticide (which is the standard susceptibility test by WHO [22]) is not sufficient information to assess the efficacy of ITNs against the malaria vectors. In fact, when the EIP and mortality rates are taken into consideration, along with multiple exposures (which is more in line with free-flying mosquitoes that are regularly host-searching and feeding), the end result is that ITNs still retain some functionality against resistant mosquitoes and work better than untreated nets. These results are in accordance with other publications $7,47,48$. We suggest an update in the way ITN efficacy and resistance are measured, as is strongly encouraged by others [5, 48,50]. For example, mosquito condition (i.e. one or more blood-feeds), age (we especially care about old mosquitoes and it has been shown previously that resistance declines with age [51]), and exposure history (multiple exposures over time since mosquitoes can encounter nets at each gonotrophic cycle) are some of the factors which combined determine the overall number of mosquitoes in a cohort potentially able to transmit malaria.

There are many unanswered questions regarding the behaviour of mosquitoes. One of these relates to feeding and biting patterns. In our model, we have assumed a constant biting rate of one bite every four days on average. We could argue that we could use a different value for each treatment. However, this was not included here since additional data is required to make appropriate estimations for a biting rate. Given that the experiment here gave access to the mosquitoes for feeding every four days, it seems more appropriate to keep this assumption. Having daily access to feeding could give more insight to estimating another biting rate. Furthermore, it would be interesting to investigate feeding and biting patterns that depend on age.

There have also been studies where it is suggested that there are parasiteinduced behavioural changes [52 55]. This could mean that the feeding and biting patterns of the mosquitoes change significantly before, during, and after the EIP. For example, mosquitoes, having survived the EIP, could bite multiple humans to complete one blood-meal, potentially transmitting the infection to more than one person. Shaw et al. found evidence that the EIP can be shortened if an infected mosquito feeds an additional time [56]; this could mean that if mosquitoes feed during the EIP and the EIP shortens, the result is more infectious bloodmeals and a larger vectorial capacity, hence an increase in malaria transmission. Therefore, it could be useful to explore other feeding patterns and bite rates.

For our calculations, we have used the Erlang distribution for the EIP. It is important to note that the mortality data were collected at $26 \pm 1^{\circ} \mathrm{C}$, whereas 
the values we obtained for the EIP come from data at $27^{\circ} \mathrm{C}$ in $[27]$. Data 496 collected at various temperatures capturing the full distribution of the EIP would be extremely useful. The EIP is affected by many factors, as shown by Ohm et al. [57], where it is emphasised that transmission models can be improved if we have a better understanding of the EIP. Some studies focus on a temperature-dependent EIP [58 60], however, given that the experiment our data were collected from was at constant temperature conditions, we have not included this here. Nevertheless, it is important to keep in mind that some factors depend on temperature in real life, so control programmes might need adjustments depending on the time of year. Incorporating temperature dependency is something else that can be explored in the future, following in the footsteps of studies like 59 and 61 .

Furthermore, to truly capture see the whole picture of malaria in Côte d'Ivoire, bringing together data for human malaria cases and other on-going control strategies with this mosquito data would help calculate the human consequences for malaria transmission and control. This could be done by constructing a modified Ross-Macdonald-type host-vector disease model [62], matching it to the data, and concurrently incorporating an age-dependent mortality rate and an Erlang-distributed EIP for the vectors.

\section{Conclusion}

In this paper, we have used a modelling framework to investigate the impact of insecticide exposure on mosquitoes and their ability to transmit malaria, along with the impact of age-dependent mortality. Firstly, our results suggest that the mortality rates increase due to insecticide exposure. Our analysis found that under a control (no insecticide exposure) scenario there would be a higher expected number of infectious bites by mosquitoes than under a treated scenario (with insecticide exposure). The vectorial capacity is substantially reduced when the mosquitoes are exposed to ITNs based on the experiment conducted, despite being resistant to the pyrethroids used on the nets. In addition, if age dependency is included in our model, the expected number of infectious bites is predicted to have a greater relative reduction by using insecticides than if we use constant mortality.

Without detailed vector data on survival with and without insecticides, this type of modelling analysis would not be possible. We strongly advocate for collection, not only of average mosquito life expectancies, but also distributions of survival for other vector-parasite systems where quantitative analyses of different interventions against the disease are desirable. We also suggest that modellers pay close attention to whether more could be done to factor in senescence into vector-borne disease strategy evaluations.

The above methodology could be easily used to check the insecticide resistance of mosquitoes from experiments using other pyrethroids and/or mosquito species, if similar experimental data on mosquito survival were available. The results could then be used to further examine how age dependency impacts the effectiveness of various interventions against mosquitoes.

516 517 518 519 520 521 522 523 524 525 526 527 528 529 530 531 532 533 534 535 536 537 538 539 


\section{Supporting information}

S1 Table Cumulative mortality data from the control treatment.

S2 Table Cumulative mortality data from the treated treatment.

S3 Table Feeding data from the control treatment.

S4 Table Feeding data from the treated treatment.

S5 Table Survival data from the control treatment.

S6 Table Survival data from the treated treatment.

S1 Fig. Proportion of total fed mosquitoes out of the alive ones for each replicate (access to foot is allowed every four days). The results from Replicate 1 are a lot different than the rest of the replicates. On the control plot (left), even at the beginning where many mosquitoes are still alive, the proportion of those that were fed is (close to) zero, and only at the very end a mosquito actually feeds. Similarly, on the right, almost all of the mosquitoes go through their lifetime without feeding.

547 548 549 550 551 552 553

S2 Fig. Cumulative deaths of mosquitoes for each replicate (recorded daily). In both plots Replicate 1 seems different to the trend followed by the other replicates, especially for the control case (left). This could be explained by the trends in S1 Fig.

S3 Fig. The probability mass function of the number of bites given the mosquito exits the extrinsic incubation period at age 15 . The average number of bites for each treatment is found in the legend box of each plot. The error bars represent the propagated uncertainty of the estimated parameters. We can see that the highest probabilities are for the smaller values of $j$ in all cases. The probability the number of bites is closer to zero is higher in the treated than in the control cases. The average number of bites is higher for the control treatment for all three cases, as expected. We also notice that for the treated case and the age-dependent functions the probability that the number of bites is equal to anything above seven is essentially zero. However, for the age-independent graph this probability goes to zero for a much higher value of $j$ which again shows how unrealistic an age-independent assumption is. S1 File Results of worst-case scenario for survival data.
554 555 556 557 558 559 560 561 562 563 564 565 566 567 568 569 


\section{Acknowledgments}

MAI was supported by EPSRC (Project Reference: EP/S022244/1) as part of the MathSys II CDT of the Warwick Mathematics Institute. MBT acknowledges funding from the Bill \& Melinda Gates Foundation, grant no. OPP1131603. The funders had no role in study design, data collection and analysis, decision to publish, or preparation of the manuscript.

We thank Raphael N'Guessan for giving access to the laboratory space, and his team for collecting the larvae in the field. We also thank N'Guessan Brou, Hermann Yapo, Koffi Henri Joel, Fernand Koffi, Hervé Koffi Kouassi, and Serge Koffi for participating in the tunnel experiments. Lastly, we thank Jacob Koella, PB's PhD supervisor, for thoughtful discussions regarding the entomological data acquisition.

\section{References}

1. Geneva: WHO. World malaria report 2020: 20 years of global progress and challenges. Licence: CC BY-NC-SA 3.0 IGO; 2020.

2. Geneva: WHO. Global technical strategy for malaria 2016-2030, 2021 update; 2021. Available from: https://www.who.int/teams/global-malaria-programme/ global-technical-strategy-for-malaria-2016-2030.

3. Bhatt S, Weiss D, Cameron E, Bisanzio D, Mappin B, Dalrymple U, et al. The effect of malaria control on Plasmodium falciparum in Africa between 2000 and 2015. Nature. 2015;526(7572):207-211.

4. Ranson H, N'guessan R, Lines J, Moiroux N, Nkuni Z, Corbel V. Pyrethroid resistance in African anopheline mosquitoes: what are the implications for malaria control? Trends in parasitology. 2011;27(2):91-98.

5. Lindsay SW, Thomas MB, Kleinschmidt I. Threats to the effectiveness of insecticide-treated bednets for malaria control: thinking beyond insecticide resistance. The Lancet Global Health. 2021;

6. Alout H, Labbé P, Chandre F, Cohuet A. Malaria vector control still matters despite insecticide resistance. Trends in parasitology. 2017;33(8):610-618.

7. Strode C, Donegan S, Garner P, Enayati AA, Hemingway J. The impact of pyrethroid resistance on the efficacy of insecticide-treated bed nets against African anopheline mosquitoes: systematic review and meta-analysis. PLoS Med. 2014;11(3):e1001619.

8. Smith DL, Perkins TA, Reiner Jr RC, Barker CM, Niu T, Chaves LF, et al. Recasting the theory of mosquito-borne pathogen transmission dynamics and control. Transactions of the Royal Society of Tropical Medicine and Hygiene. 2014;108(4):185-197.

9. Dietz K. The estimation of the basic reproduction number for infectious diseases. Statistical methods in medical research. 1993;2(1):23-41.

10. Garrett-Jones C. Prognosis for interruption of malaria transmission through assessment of the mosquito's vectorial capacity. Nature. 1964;204(4964):11731175. 
11. Smith DL, Battle KE, Hay SI, Barker CM, Scott TW, McKenzie FE. Ross, Macdonald, and a theory for the dynamics and control of mosquito-transmitted pathogens. PLoS pathog. 2012;8(4):e1002588.

12. Styer LM, Carey JR, Wang JL, Scott TW. Mosquitoes do senesce: departure from the paradigm of constant mortality. The American journal of tropical medicine and hygiene. 2007;76(1):111-117.

13. Dawes EJ, Churcher TS, Zhuang S, Sinden RE, Basáñez MG. Anopheles mortality is both age-and Plasmodium-density dependent: implications for malaria transmission. Malaria journal. 2009;8(1):1-16.

14. Bellan SE. The importance of age dependent mortality and the extrinsic incubation period in models of mosquito-borne disease transmission and control. PLoS One. 2010;5(4):e10165.

15. Novoseltsev VN, Michalski AI, Novoseltseva JA, Yashin AI, Carey JR, Ellis AM. An age-structured extension to the vectorial capacity model. PLoS One. 2012;7(6):e39479.

16. Ryan SJ, Ben-Horin T, Johnson LR. Malaria control and senescence: the importance of accounting for the pace and shape of aging in wild mosquitoes. Ecosphere. 2015;6(9):1-13.

17. Brand SP, Rock KS, Keeling MJ. The interaction between vector life history and short vector life in vector-borne disease transmission and control. PLoS computational biology. 2016;12(4):e1004837.

18. Assouho KF, Adja AM, Guindo-Coulibaly N, Tia E, Kouadio AM, Zoh DD, et al. Vectorial transmission of malaria in major districts of Côte d'Ivoire. Journal of medical entomology. 2020;57(3):908-914.

19. Ashley EA, Phyo AP, Woodrow CJ. Malaria. The Lancet. 2018;391(10130):16081621.

20. Oumbouke WA, Pignatelli P, Barreaux AM, Tia IZ, Koffi AA, Alou LPA, et al. Fine scale spatial investigation of multiple insecticide resistance and underlying target-site and metabolic mechanisms in Anopheles gambiae in central Côte d'Ivoire. Scientific reports. 2020;10(1):1-13.

21. Kulma K, Saddler A, Koella JC. Effects of age and larval nutrition on phenotypic expression of insecticide-resistance in Anopheles mosquitoes. PLoS One. 2013;8(3):e58322.

22. WHO, et al.. Test procedures for insecticide resistance monitoring in malaria vector mosquitoes; 2016.

23. Catano-Lopez A, Rojas-Diaz D, Laniado H, Arboleda-Sánchez S, Puerta-Yepes ME, Lizarralde-Bejarano DP. An alternative model to explain the vectorial capacity using as example Aedes aegypti case in dengue transmission. Heliyon. 2019;5(10):e02577.

24. Massad E, Coutinho FAB. Vectorial capacity, basic reproduction number, force of infection and all that: formal notation to complete and adjust their classical concepts and equations. Memórias do Instituto Oswaldo Cruz. 2012;107(4):564567.

25. Moller-Jacobs LL, Murdock CC, Thomas MB. Capacity of mosquitoes to transmit malaria depends on larval environment. Parasites \& vectors. 2014;7(1):593. 
26. CDC. CDC - Malaria - About Malaria - Biology; 2020. Available from: https: //www.cdc.gov/malaria/about/biology/index.html.

27. Stopard IJ, Churcher TS, Lambert B. Estimating the extrinsic incubation period of malaria using a mechanistic model of sporogony. PLoS Computational Biology. $2021 ; 17$.

28. Shapiro LL, Whitehead SA, Thomas MB. Quantifying the effects of temperature on mosquito and parasite traits that determine the transmission potential of human malaria. PLoS biology. 2017;15(10):e2003489.

29. Shapiro LL, Murdock CC, Jacobs GR, Thomas RJ, Thomas MB. Larval food quantity affects the capacity of adult mosquitoes to transmit human malaria. Proceedings of the Royal Society B: Biological Sciences. 2016;283(1834):20160298.

30. Bompard A, Da DF, Yerbanga SR, Morlais I, Awono-Ambéné PH, Dabiré RK, et al. High Plasmodium infection intensity in naturally infected malaria vectors in Africa. International Journal for Parasitology. 2020;50(12):985-996.

31. Murdock C, Sternberg E, Thomas M. Malaria transmission potential could be reduced with current and future climate change. Sci Rep. 6: 27771; 2016.

32. Gubbins S, Carpenter S, Baylis M, Wood JL, Mellor PS. Assessing the risk of bluetongue to UK livestock: uncertainty and sensitivity analyses of a temperaturedependent model for the basic reproduction number. Journal of the Royal Society Interface. 2008;5(20):363-371.

33. Wearing HJ, Rohani P, Keeling MJ. Appropriate models for the management of infectious diseases. PLoS Med. 2005;2(7):e174.

34. Rock KS, Torr SJ, Lumbala C, Keeling MJ. Quantitative evaluation of the strategy to eliminate human African trypanosomiasis in the Democratic Republic of Congo. Parasites \& vectors. 2015;8(1):1-13.

35. Childs LM, Prosper OF. The impact of within-vector parasite development on the extrinsic incubation period. Royal Society open science. 2020;7(10):192173.

36. Chan M, Johansson MA. The incubation periods of dengue viruses. PloS one. 2012;7(11):e50972.

37. Ibe OC. 1 - Basic Concepts in Probability. In: Ibe OC, editor. Markov Processes for Stochastic Modeling (Second Edition). second edition ed. Oxford: Elsevier; 2013. p. 1-27. Available from: https://www.sciencedirect.com/science/article/ pii/B9780124077959000013.

38. Rock K, Wood D, Keeling M. Age-and bite-structured models for vector-borne diseases. Epidemics. 2015;12:20-29.

39. Rodríguez G. Lecture Notes on Generalized Linear Models; 2007. Available from: https://data.princeton.edu/wws509/notes/.

40. Bezanson J, Edelman A, Karpinski S, Shah VB. Julia: A fresh approach to numerical computing. SIAM Review. 2017;59(1):65-98. doi:10.1137/141000671.

41. Giordano M. Uncertainty propagation with functionally correlated quantities. ArXiv e-prints. 2016;

42. Hahn T. Cuba - a library for multidimensional numerical integration. Computer Physics Communications. 2005;168(2):78-95.

43. Hahn T. Concurrent cuba. Computer Physics Communications. 2016;207:341-349. 
44. Luz PM, Struchiner CJ, Galvani AP. Modeling transmission dynamics and control of vector-borne neglected tropical diseases. PLoS Negl Trop Dis. 2010;4(10):e761.

45. Hollingsworth TD, Pulliam JR, Funk S, Truscott JE, Isham V, Lloyd AL. Seven challenges for modelling indirect transmission: Vector-borne diseases, macroparasites and neglected tropical diseases. Epidemics. 2015;10:16-20.

46. Clements A, Paterson G. The analysis of mortality and survival rates in wild populations of mosquitoes. Journal of applied ecology. 1981; p. 373-399.

47. Glunt KD, Coetzee M, Huijben S, Koffi AA, Lynch PA, N'Guessan R, et al. Empirical and theoretical investigation into the potential impacts of insecticide resistance on the effectiveness of insecticide-treated bed nets. Evolutionary applications. 2018;11(4):431-441.

48. Viana M, Hughes A, Matthiopoulos J, Ranson H, Ferguson HM. Delayed mortality effects cut the malaria transmission potential of insecticide-resistant mosquitoes. Proceedings of the National Academy of Sciences. 2016;113(32):8975-8980.

49. Collins E, Vaselli NM, Sylla M, Beavogui AH, Orsborne J, Lawrence G, et al. The relationship between insecticide resistance, mosquito age and malaria prevalence in Anopheles gambiae sl from Guinea. Scientific reports. 2019;9(1):1-12.

50. Thomas MB, Read AF. The threat (or not) of insecticide resistance for malaria control. Proceedings of the National Academy of Sciences. 2016;113(32):8900-8902.

51. Glunt KD, Thomas MB, Read AF. The effects of age, exposure history and malaria infection on the susceptibility of Anopheles mosquitoes to low concentrations of pyrethroid. PLoS One. 2011;6(9):e24968.

52. Anderson RA, Koellaf J, Hurd H. The effect of Plasmodium yoelii nigeriensis infection on the feeding persistence of Anopheles stephensi Liston throughout the sporogonic cycle. Proceedings of the Royal Society of London Series B: Biological Sciences. 1999;266(1430):1729-1733.

53. Wekesa JW, Copeland RS, Mwangi RW. Effect of Plasmodium falciparum on blood feeding behavior of naturally infected Anopheles mosquitoes in western Kenya. The American journal of tropical medicine and hygiene. 1992;47(4):484-488.

54. Cator LJ, George J, Blanford S, Murdock CC, Baker TC, Read AF, et al. 'Manipulation'without the parasite: altered feeding behaviour of mosquitoes is not dependent on infection with malaria parasites. Proceedings of the Royal Society B: Biological Sciences. 2013;280(1763):20130711.

55. Rossignol P, Ribeiro J, Spielman A. Increased intradermal probing time in sporozoite-infected mosquitoes. The American journal of tropical medicine and hygiene. 1984;33(1):17-20.

56. Shaw WR, Holmdahl IE, Itoe MA, Werling K, Marquette M, Paton DG, et al. Multiple blood feeding in mosquitoes shortens the Plasmodium falciparum incubation period and increases malaria transmission potential. PLoS Pathogens. 2020;16(12):e1009131.

57. Ohm JR, Baldini F, Barreaux P, Lefevre T, Lynch PA, Suh E, et al. Rethinking the extrinsic incubation period of malaria parasites. Parasites \& vectors. 2018;11(1):19.

58. Kamiya T, Greischar MA, Wadhawan K, Gilbert B, Paaijmans K, Mideo N. Temperature-dependent variation in the extrinsic incubation period elevates the risk of vector-borne disease emergence. Epidemics. 2020;30:100382. 
59. Paaijmans KP, Blanford S, Chan BH, Thomas MB. Warmer temperatures reduce the vectorial capacity of malaria mosquitoes. Biology letters. 2012;8(3):465-468.

60. Chua $\mathrm{T}$, et al. Modelling the effect of temperature change on the extrinsic incubation period and reproductive number of Plasmodium falciparum in Malaysia. Trop Biomed. 2012;29(1):121-8.

61. Suh E, Grossman MK, Waite JL, Dennington NL, Sherrard-Smith E, Churcher TS, et al. The influence of feeding behaviour and temperature on the capacity of mosquitoes to transmit malaria. Nature Ecology \& Evolution. 2020; p. 1-12.

62. Reiner Jr RC, Perkins TA, Barker CM, Niu T, Chaves LF, Ellis AM, et al. A systematic review of mathematical models of mosquito-borne pathogen transmission: 1970-2010. Journal of The Royal Society Interface. 2013;10(81):20120921. 